\title{
MULTISTAGE CLASSIFICATION OF DIABETIC RETINOPATHY USING FUZZY- NEURAL NETWORK CLASSIFIER
}

\author{
Deepthi K Prasad $^{1}$, L. Vibha ${ }^{2}$ and K.R. Venugopal ${ }^{3}$ \\ ${ }^{1,2}$ Department of Computer Science Engineering, B N M Institute of Technology, India \\ ${ }^{3}$ Department of Computer Science Engineering, University Visvesvaraya College of Engineering, India
}

\begin{abstract}
Diabetic Retinopathy $(\mathrm{DR})$ is complicated disorder in human retina which is affected due to an increasing amount of insulin in blood that results in vision impairment. Early detection of DR is used to support the patients to prevent blindness and to be aware of this disease. This paper proposes a novel technique for detecting DR using hybrid classifiers. It includes pre-processing of the image, segmentation of region of interest, feature extraction and classification. Retinal structures like microaneurysms, exudates, hemorrhages and blood vessels are segmented. Classification is performed with integration of Fuzzy logical System and Neural Network (NN) which improves the accuracy of classification. Experimentation is carried out with the MESSIDOR data set. Results are compared against various performance metrics like accuracy, sensitivity and specificity. An accuracy close to 100 percent and low average error rate of 0.012 are obtained using the proposed method. The results obtained are encouraging.
\end{abstract}

Keywords:

Diabetic Retinopathy, Hybrid Classifier, Visual Impairment, Fundus Images, Classification, Fuzzy Neural Network

\section{INTRODUCTION}

DR is a micro vascular complication which is an eye related disease among people with diabetes and is the main reason for visual impairment in patients from 25 to 75 years of age [1]. According to the survey of World Health Organization (WHO) [2] about 285 million people are visually impaired and blind person in world wide. In 2030 [3], the total number of diabetic patients will at least reach to 366 million. The probability of affected people is increased $25 \%$, but timely treatment and early detection reduce the anxiety of the patients thereby preventing severe vision loss as well as blindness.

DR has no symptoms in initial stages. The Fig.1(a) represents the healthy retina with normal macula, blood vessels and optic nerve. The Fig.1(b) illustrates the DR with the presence of cotton wool spots, Microaneurysms, edema, exudates and neovascularization. Fundus images are essential in DR to monitor retinal abnormalities. The efficiency is dependent on accurate capturing of fundus image and reliable of the image processing techniques [5] - [8]. Image processing techniques are preprocessing, segmentation, feature extraction and classification.

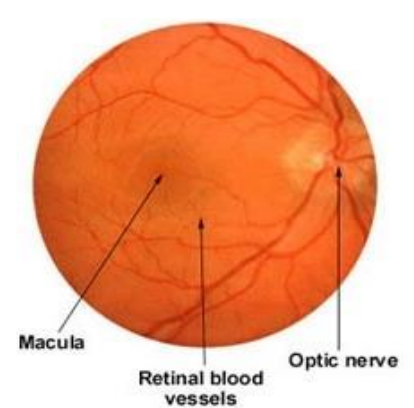

(a)

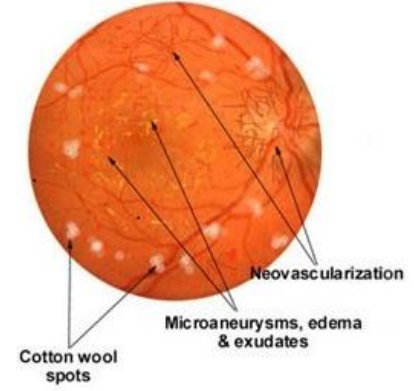

(b)
Fig.1. (a) Normal Retina, (b) Diabetic Retinopathy (DR)

Pre-processing is one of the significant steps for removing variations in the retinal fundus image and it enhances image quality and contrast of fundus image. Several pre-processing techniques are used which includes noise removal, Normalization, intensity conversion, binarization, morphological operations, etc. Segmentation is the task of dividing the images into several regions for easily analyzing images. Segmentation is performed with illumination correlation Gabor filtering, Adaptive threshold, Edge detection and Hough transform. Feature extraction is an important process for classifying the images into different categories. The different types of features are computed as statistical measure, colour model, histogram, Fourier spectra, Principal Component Analysis (PCA), fractal dimension and textual analysis. Classification is a process that helps to classify the images based on selected features. The various types of classifiers are Random Forest, Genetic Algorithm (GA), knearest neighbor, Support Vector Machine (SVM), Naïve Bayes, Decision tree, Fuzzy Set Theory and Neural Networks (NN).

The contribution of the proposed work is summarized as follows:

- Designed better pre-processing techniques by RGB to Gray scale, Noise removal, Contrast enhancement and Morphological processing which improves image quality

- Retinal structures are segmented based on microaneurysms, exudates, hemorrhages and blood vessels.

- Fuzzy logical system and NN is applied for classifying the DR into multistage as normal, mild, moderate, severe and PDR

DR occurs due to elevations in blood sugar level that would damage the blood vessels of retina. DR can gradually become more serious and affect vision causes blindness, if not early treated. The important feature components of DR are Microaneurysms, hemorrhages, exudates, blood vessels [9] [18] given in Table.1. 
Table.1. DR Features

\begin{tabular}{|c|c|}
\hline $\begin{array}{c}\text { Retinal } \\
\text { Structure }\end{array}$ & Description \\
\hline Microaneurysms & $\begin{array}{c}\text { Microaneurysms represent most } \\
\text { fundamental observable indicator of retinal } \\
\text { damage. They are small, round in shape and } \\
\text { dark red dots appear on the retinal layers. }\end{array}$ \\
\hline Hemorrhages & $\begin{array}{c}\text { They are similar to microaneurysms that } \\
\text { will appear beneath the layers of retina. Two } \\
\text { types: dot hemorrhages and blot hemorrhages. }\end{array}$ \\
\hline Hard Exudates & $\begin{array}{c}\text { Exudates have high intensity compared to } \\
\text { the rest and appear as yellow spots with sharp } \\
\text { margins. HE are not regular in shape and are } \\
\text { usually located near to Microaneurysms. }\end{array}$ \\
\hline $\begin{array}{c}\text { Soft exudates } \\
\text { (Cotton Wood } \\
\text { Spots) }\end{array}$ & $\begin{array}{c}\text { It reduces blood circulation to retina that } \\
\text { causes ischemia of Retinal Nerve Fiber Layer } \\
\text { (RNFL). }\end{array}$ \\
\hline
\end{tabular}

DR is separated into two types with respect to new vessels growth: Non-Proliferative Diabetic Retinopathy (NPDR) and Proliferative Diabetic Retinopathy (PDR). These types [4] of retinopathy are graded into four stages based on severity of DR feature.

- Mild NPDR: It is an early stage of disease in which symptoms will be mild or nonexistent. NPDR is also known as background retinopathy. It is characterized by the presence of dot as well as blotch hemorrhages and microaneurysms in the retina. In this stage microaneurysms are areas of swelling tiny blood vessels that caused by retina structure

- Moderate NPDR: It is a second stage of DR, in this stage some of small blood vessels are blocked that leads to decrease the supply of oxygen and nutrients to certain areas of retina.

- Severe NPDR: In this stage significant numbers of small blood vessels are blocked which results in more areas of retina being depressed of oxygen and nourishment.

- PDR: It is more advance stage of disease. At this stage, new blood vessels are abnormally developed in retina, called neovascularization. These vessels are bleeding into the retinal that causes vision loss

These different stages of DR are depicted in Fig.2.

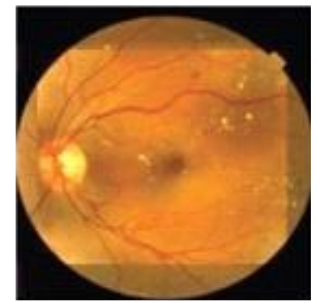

(a) Mild NPDR

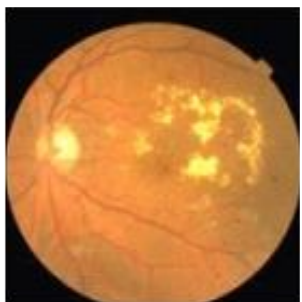

(b) Moderate NPDR

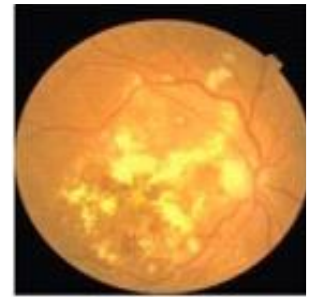

(c) Severe NPDR

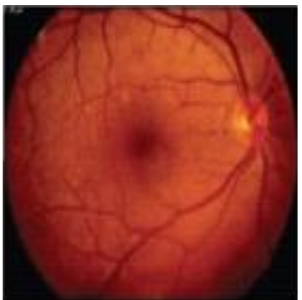

(d) PDR
Fig.2. Different stages of DR

The structure of the work is described below. Section 2 explores the Literature work, Section 3 incorporates description of the proposed work, section 4 deals with experimental results along with simulation environment and finally section 5 concludes the proposed paper work.

\section{LITERATURE REVIEW}

Retinal fundus images are mainly used to detect and classify retinal diseases in earlier stage. Many researchers have proposed different algorithms and techniques for classifying DR into various stages.

\subsection{PREPROCESSING}

The authors of [9] discussed on an automatic detection of microaneurysms in retina. Initially, pre-processing was performed with extraction of green channel, contrast enhancement and histogram equalization. These pre-processing processes are not capable to provide noise removal completely for further process. To overcome this problem, de-noising was concentrated in this work [10]. Median filter was used for removing salt and pepper noise without fading sharp edges. Low frequency information in background has not been removed significantly by this filtering. To improve the blurring, size and non-clarity problems, gray scale model were designed with separation of red, blue, green channels. Histogram equalization is performed after filtering process for contrast enhancement [11]. Fuzzy pre-processing techniques were used for enhancing the image quality [12]. It was performed with fuzzy filtering, fuzzy histogram equalization and fuzzy median filtering. Here, filtering process was not able to provide quality image. Further, pre-processing was applied by green channel extraction, and then Robust Spatial Kernel FCM (RSKFCM) method was designed for eliminating optic disk. RSKFCM estimates the distance value based on threshold function with support of local neighbourhood pixel and kernel distance function. This process provides improper classification since it does not able to remove noise in pre-processing.

\subsection{SEGMENTATION}

After completion of pre-processing, segmentation is important for improving accuracy, specificity, speed and sensitivity Authors of [15] concentrated on separation of blood vessels using two dimensional Gabor wavelet and Fuzzy Mathematical Morphology (FMM). Then, pipeline analysis was applied to estimate background strength of FMM. Accuracy becomes low due to presence of some noise particles. In order to improve the accuracy, projection based segmentation was proposed with two 
stages as pre-handling, exudates segmentation. Pre-handling was utilized through noise removal and contrast enhancement. Exudates segmentation was used to select projection values based on threshold function. However, it takes a lot of time to find exudates and also low accuracy in finding exudates [16]. Genetic Algorithm (GA) was designed for locating exudates and blood vessels with high accuracy and low error rate [17]. GA randomly generates the set of possible solution by using operators as reproduction, crossover and mutation. However, the process of GA is difficult to choose best fitness value and also consumes more time to computation.

\subsection{EXTRACTION OF FEATURES}

The aim of this phase is to minimize the extent of resources necessary to describe huge data set. In [9], feature extraction was used to seperate the optic disk and blood vessels. Statistical features are extracted from microaneurysms with support of standard deviation. In this feature extraction is only focused on microaneurysms so it is not able to identify the severe stages of DR. In [10] feature extraction was used for improving the classification process. Features such as exudates, blood vessels, microaneurysms and optic discs were extracted by using morphological operations. It consumes high time and also not effective for noise removal. Automatic Screening process was designed for detecting effective features in retinal fundus image [11]. Retinal microaneurysms, exudates, cotton wool spots and hemorrhages are selected for identifying NPDR and PDR. Twostep hierarchal binary classification method was designed for avoiding false positive in feature extraction [12]. In this process, top thirty features are considered from a set of seventy eight features for analyzing retinal lesions. Feature ranking algorithm is designed for extracting the features with support of AdaBoost which is used to estimate feature weights.

\subsection{CLASSIFICATION}

Several classification techniques have been proposed to classify DR. Most of the researchers have concentrated on machine learning algorithms to solve classification problems. SVM was designed for computerized detection of DR in retinal images [9]. SVM classifies the various stages of DR by constructing data linear hyper plane with linear kernel function. This paper is focused on microaneurysms which are effective for classifying mild stage and it is not able to provide accurate result in severe stage. Further, combination of SVM and K-NN classifiers was proposed for improving classification accuracy level [11]. K-NN only supports well defined training data. Three stages of algorithm were designed for automatic classification and detection [12]. Two-step binary classification was used for automatic detection. Different classification methods are proposed as SVM, KNN, Gaussian Mixture Model (GMM) and ADABOOST for analyzing retinopathy lesions from non-lesions. However, this process consumes more time to classify since it uses many classification techniques. Authors of [14] have concentrated on classification and detection of exudates by using automatic techniques. Classification was performed with sequential learning algorithm, called Meta-Cognitive Neural Network (McNN). This McNN comprises of input, hidden and output layers. All features are fed into hidden layer which is performed with Gaussian and linear activation function. Fuzzy image processing technique was developed to automatically detect the DR and maculopathy in retinal fundus images [13]. The proposed method includes image capturing, pre-processing (retinal structure localization), attribute extraction and classification. Classification was performed with k-Nearest Neighbour, and Naïve-Bayes classifiers.

\section{PROPOSED SYSTEM}

The proposed work produces a new solution for DR detection based on severity of stages as normal, mild, moderate, severe and PDR. Initially, Input image is considered from Messidor database for DR detection process. Preprocessing is an important process to enhance quality of image for accurate classification that consists of following steps such as RGB to grey scale conversion, noise removal, contrast enhancement and morphological processing. Then, segmentation is used to isolate the relevant parts of retinal image into microaneurysms, exudates, hemorrhages and blood vessels. Further, feature extraction is processed with three types of features as texture, shape and statistical features which is important to provide best set of features that is effectively used for classification with low error rate. Finally, classification is performed by using hybrid classifier which is combination of NN and fuzzy logical system. This hybrid classification technique improves the classification process based on severity of disease. The Fig. 3 represents architecture of the proposed system.

\subsection{PRE-PROCESSING}

The purpose of pre-processing is to extract all possible regions from input retinal image without any background errors. The steps involved in pre-processing stage are as follows:

- RGB to Gray Scale

- Noise Removal

- Contrast Enhancement

- Morphological processing

\subsubsection{RGB to Gray Scale:}

The original input image is converted into gray scale image which gives only intensity information after Saturation and Hue are removed from the color image. The conversion of RGB to gray scale is done by using Eq.(1),

$$
I_{Y}=0.333 F_{R}+0.5 F_{G}+0.1666 F_{B}
$$

where, $F_{R}, F_{G}$ and $F_{B}$ represent the intensity of $R, G, B$ components and $I_{Y}$ denotes intensity of equivalent gray level image of RGB.

\subsubsection{Noise Removal:}

The noise is reduced by using Wavelet Transform Thresholding which is used to enhance the relevant image content without noise factors. WTT has two types are Hard Thresholding (HT) and Soft Thresholding (ST) as follows,

$$
H T(X)=\theta_{H}\left(y, \lambda_{t}\right)= \begin{cases}0 & \text { if }|y|<\lambda_{t} \\ y & \text { otherwise }\end{cases}
$$

and

$$
S T=\theta_{H}\left(y, \lambda_{t}\right)=\operatorname{sign}(y)\left(|y|-\lambda_{t}\right)
$$


Here $\lambda_{t}$ represents universal Threshold which is estimated using the Eq.(2):

$$
\lambda_{t}=\sigma \sqrt{2 \log N}
$$

where, $\sigma$ denotes noise variance and $N$ represents size of the image

\subsubsection{Contrast Enhancement:}

The contrast of retinal background is improved to accommodate for segmentation at a later stage. Local contrast enhancement process is applied for allocating pixel values around local mean. In small running window $W$ the center pixel $P$ is changed into new value $P_{N}$. The value of $P_{N}$ is calculated as follows,

$$
P_{N}=255\left(\frac{\left[\phi_{W}(p)-\phi_{W}(\operatorname{Min})\right]}{\left[\phi_{W}(\operatorname{Max})-\phi_{W}(\operatorname{Min})\right]}\right) .
$$

Here,

$$
\phi_{W}(p)=\left[1+\exp \left(\frac{\mu_{W}-P}{\sigma_{W}}\right)\right]
$$

where, min and max represents minimum and maximum value of whole image $\mu_{W}$ denotes local window mean and $\sigma_{W}$ represents local window standard deviation.

\subsubsection{Morphological Processing:}

This involves various morphological operations for detecting blood vessels. The morphological operations in mathematical form with erosion and dilation are as follows

- Erosion: In erosion image $I_{i}$ is symbolized by the structural element $A_{i}$ which is quantified by set of operation such as,

$$
I_{i} \ominus A_{i}=\left\{p \in z^{2} \mid(p+q) \in I_{i}\right\}
$$

for every q $\in A_{i}$.

- Dilation: In dilation image $I_{i}$ is symbolized by the structural element $A_{i}$ which is quantified by set of operation such as,

$$
I_{i} \oplus A_{i}=\left\{(p+q) \mid p \in I_{i}, q \in A_{i}\right\}
$$

\subsection{SEGMENTATION}

Segmentation is an important task for finding DR in effective manner. It uses the enhanced background image, which divides into low and high density areas for segmentation of microaneurysms, hemorrhages, exudates, blood vessels separately. Intensity based properties are used for segmenting the different DR retinal structure [19]. Standard deviation $(\sigma)$ and arithmetic mean $(\mu)$ values are calculated on entire image. Then threshold value $T$ is assigned for segmenting each retinal structure.

$$
\begin{gathered}
\mu=\frac{1}{N} \sum_{i=1}^{N} x_{i}(7) \\
\sigma=\sqrt{\frac{1}{N} \sum_{i=1}^{N}\left(x_{i}-\mu\right)^{2}}
\end{gathered}
$$

where, $N$ represents total number of pixels, $x_{i}$ denotes pixel values of entire image. The Fig.4 illustrates the segmentation with (a)
Normal image, (b) Blood vessels segmentation, (c) exudates segmentation and (d) microaneurysms, hemorrhages segmentation.

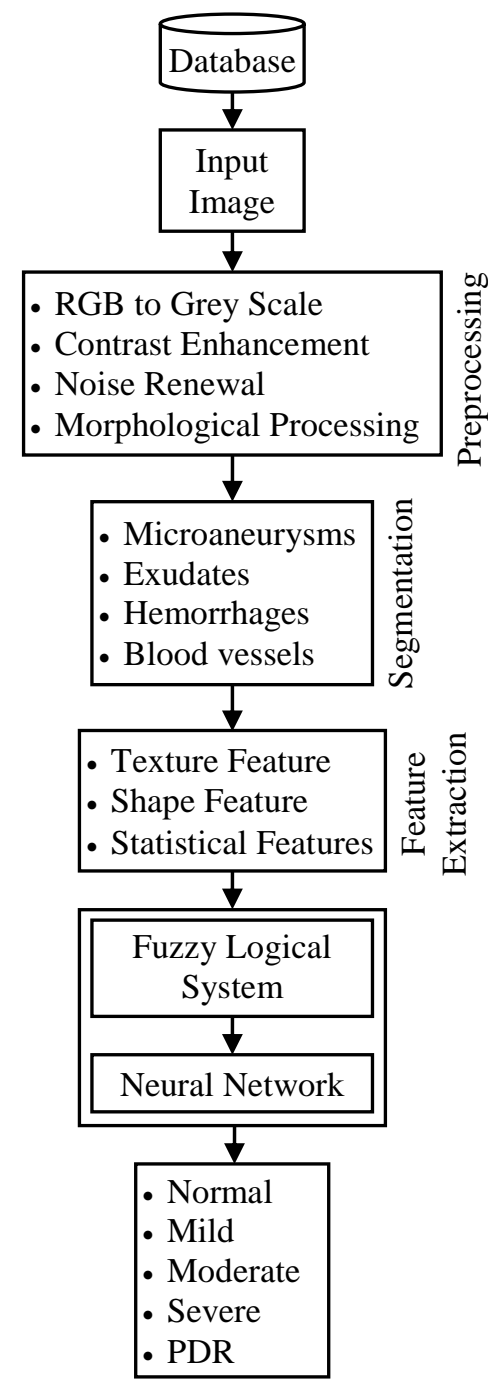

Fig.3. Architecture and Modelling

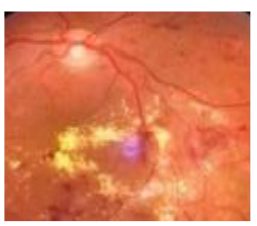

(a)

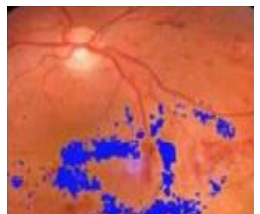

(c)

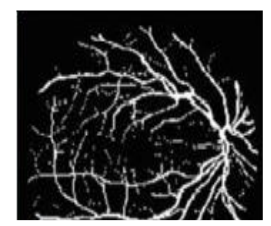

(b)

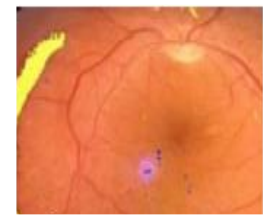

(d)
Fig.4. Segmentation Process (a) Normal image, (b) Blood vessels segmentation, (c) exudates segmentation and (d) microaneurysms, hemorrhages segmentation 


\subsection{EXTRACTION OF ATTRIBUTES}

Attribute extraction is a vital process for classification that reduces the complication of classification process. The $\mathrm{g}$ three types of features used for extraction process as texture features, intensity features and shape features.

\subsubsection{Texture Feature Extraction:}

Texture features are extracted by using texture descriptor Local Binary Pattern (LBP). LBP is computationally efficient which is robust against various illuminations of gray levels and it is simple yet efficient to assess fundus images. LBP captures small micro-patterns very easily. So it is suitable for detecting features of microaneurysms, hemorrhages, exudates, blood vessels. The mathematical equation of LBP is as follows,

$$
\begin{gathered}
L B P_{p, R}=\sum_{p=1}^{p-1} S\left[G_{p}-G_{C}\right] 2^{p}, \\
S(X)= \begin{cases}1 & \text { if } X \geq 0 \\
0 & \text { if } X<0\end{cases}
\end{gathered}
$$

where, $G_{c}$ and $G_{P}$ represent the grey values of central and neighborhood pixel respectively, $P$ indicates sample numbers on the symmetric circular neighborhood of radius. $2^{P}$ denotes the binary patterns that are produced for each neighborhood.

\subsubsection{Shape Features:}

Shape features are important since retinal structures have different shapes.

- Circularity: Circularity is denotes as degree to which the particle is similar to circle, taking into consideration of smoothness of perimeter. It is measured particle form with a function of area $(A)$ and perimeter $(P)$,

$$
C_{\text {Circle }}=\frac{4 \pi A}{p^{2}}
$$

- Aspect ratio (AR): Aspect ratio has been used historically to classify general form of particles (for instance, equant, fibrous or acicular). The aspect ratio specifies the largest dimension $d_{\max }$ and smallest dimension $d_{\min }$.

$$
A R=\frac{d_{\min }}{d_{\max }}
$$

- Elongation Shape factor: It is distinct as the square root of the two moments $(I)$ of the pixel with a particular principal axes.

$$
\text { Elong }=\sqrt{\frac{i_{2}}{i_{1}}}
$$

- Compactness Shape factor: It is compactness measure of shape also known as shape factor. It is a function of a circle of equal area $A$ and polar moment ( $i$ ) of a particle.

$$
\text { Comp }=\frac{A^{2}}{4 \pi \sqrt{i_{1}^{2}+i_{2}^{2}}}
$$

\subsubsection{Statistical Features:}

Statistical features are also known as disease based features which are area and entropy. These features are significant for effectively analyze the retinal structure
- Area: Retinal structure area is calculated based on severity of disease. This feature is efficient to locate an object in two dimensional image plane. The area is measured in pixels and indicates the relative size of object as follows:

$$
a(i, j)=\frac{1}{x-y} \sum_{x=0}^{X-J} \sum_{y=0}^{Y-J} b_{i}(i-x, j-y)
$$

where $X, Y$ represents the regions of every $(i, j)$ point of the image

- Entropy: It describes the amount of uncertainty in the texture of input image. To measure the homogeneity of segment region, Gray-level image entropy (GIE) is estimated as

$$
G I E=H[P(g)]-\sum_{j=1}^{J}\left[P_{j}(g)\right]
$$

\subsection{CLASSIFICATION}

Classification is a decision making process which is used to classify the images into different stages based on their features. Classification mechanism analyzes the numerical properties of various image features and categorizes it. Fuzzy logical set and NN (Neuro-fuzzy) are used for DR detection and classification of retinal image. The proposed hybrid classifier uses a learning method for assessing training data for finding a best way to classify the DR images into normal, mild, moderate, severe, and PDR.

Neuro-fuzzy system is depending on fuzzy system which is trained by learning algorithm from neural network process. It can be viewed as a feed forward neural network with three layers. The first layer denotes the input variables, second layer represents fuzzy rules and their third layer denotes output variables. The proposed Neuro-fuzzy model is employed to produce accurate results when additional features are added to the input parameters. The key pros of this model are its capability to learn and its simplicity of implementation. The Neuro-Fuzzy classifier takes the features that are extracted as its input and classifies the retinal fundus images into multistage based on their severity. The Fig.5 represents the Neuro-fuzzy system and its layers performance.

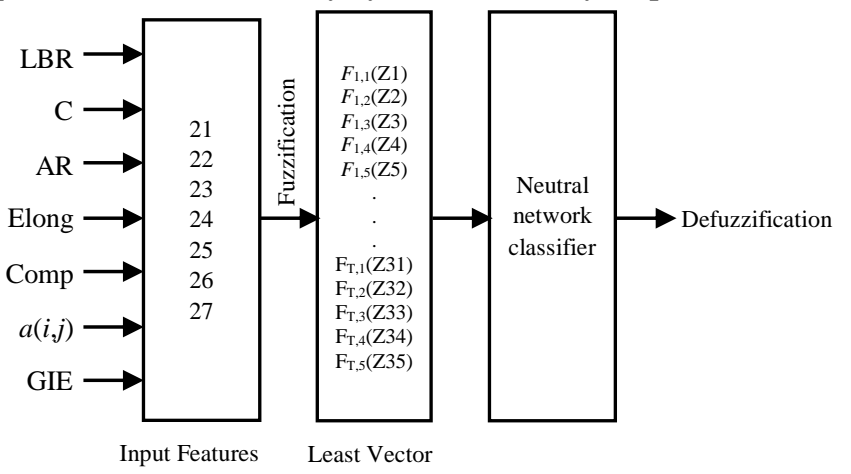

Fig.5. Neuro-Fuzzy System

The feature values which are taken as input are fuzzified with support of membership functions. Membership function extracts the unknown mutually connected information from the features towards classes. This membership matrix is figured with seven rows and five columns, in which rows are equal to number of features and columns is represented as number of stages. This matrix is converted into vector value and then it feeding into NN 
classifier. Max operation is performed in defuzzification process which carries out the output value into multistage as normal or mild or moderate or severe or PDR.

\section{EXPERIMENTAL EVALUATION}

This section discusses about outcome of the proposed work. This section is comprised with sub-sections as database description, performance metrics, result discussion and comparative analysis. With this result it is clear that the proposed work is used for accurately classifying and detecting the multistage DR in fundus image. The comparative analysis is done with previous classification approaches. The Table. 2 discusses about problems existed in state-of-the-art, which is tackled in the proposed work by concentrating better pre-processing, segmentation, feature extraction and classification as well as analyzing it with the proposed comparative results.

Table.2. Problems of State-of-the-Art

\begin{tabular}{|c|c|}
\hline State-of-the-art & Problems \\
\hline $\begin{array}{c}\text { Three stage } \\
\text { algorithm [12] }\end{array}$ & $\begin{array}{l}\text { Improper classification due to use of } \\
\text { many classifiers }\end{array}$ \\
\hline GA [17] & $\begin{array}{l}\text { Difficult to choose best fitness value } \\
\text { High computation time }\end{array}$ \\
\hline $\begin{array}{c}\text { 2D Gabor } \\
\text { Wavelet [15] }\end{array}$ & $\begin{array}{c}\text { Low accuracy } \\
\text { It is not able to effectively remove } \\
\text { noise particles }\end{array}$ \\
\hline
\end{tabular}

\subsection{MESSIDOR DATABASE}

Messidor database is used in the proposed work. It consists of 1,200 fundus images with three distinct resolution ranges such as $1400 \times 960,2240 \times 1488,2304 \times 1536$. Here, 800 images are obtained with pupil dilation and 400 images are have not undergone dilation.

\subsection{PERFORMANCE METRICS}

Performance metrics are significant for calculating pixel-pixel based on four values such as True Positive $(T P)$, True Negative $(T N)$, False positive $(F P)$ and False Negative $(F N)$. It is defined as,

$T P$ - Positive pixels correctly labeled as positive.

$F P$ - Negative pixels incorrectly labeled as positive.

$F N$ - Positive pixels incorrectly labeled as negative.

$T N$ - Negative pixels incorrectly labeled as negative.

The most significant metrics are sensitivity, specificity which improves the accuracy measurement of diagnosis methods.

\subsubsection{Sensitivity:}

Sensitivity is defined as the amount of abnormal result.

$$
\text { Sensitivity }(\%)=\frac{T P}{T P+F N} \times 100
$$

\subsubsection{Specificity:}

Specificity is defined as proportion of actual negative (normal) result.

$$
\operatorname{Specificity}(\%)=\frac{T N}{T N+F P} \times 100
$$

\subsubsection{Accuracy:}

Accuracy is one of the significant metric which can be used to evaluate the overall system performance. Accuracy is computed by following equation,

$$
\operatorname{Accuracy}(\%)=\frac{T N+T P}{T N+T P+F P+F N} \times 100 \%
$$

\subsection{RESULT AND DISCUSSIONS}

This section discusses about the experimental result of every stage such as pre-processing, segmentation, feature extraction and classification. The Fig.7 illustrates pre-processing results of RGB to Grey scale conversion, noise removal, contrast enhancement and morphological processing.

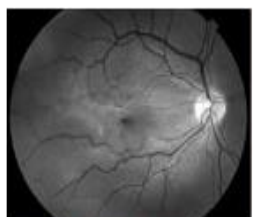

(a)

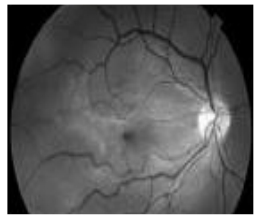

(c)

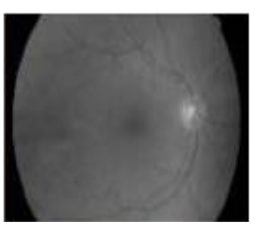

(b)

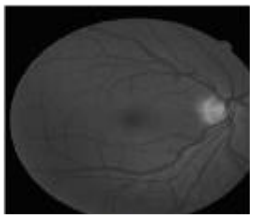

(d)
Fig.7. Pre-processing Results: (a) RGB to Gray (b) Noise Removal (c) Contrast Enhancement and (d) Morphological Processing

The segmentation process involves intensity based operations for segmenting retinal structures such as microaneurysms, hemorrhages, exudates, blood vessels separately This segmentation results supports to improve the accuracy of overall result. Results of segmentation are depicted in Fig.8.

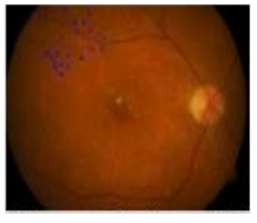

(a)

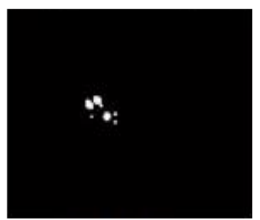

(c)

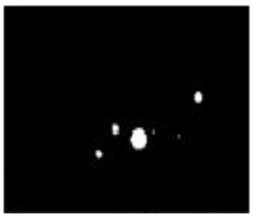

(b)

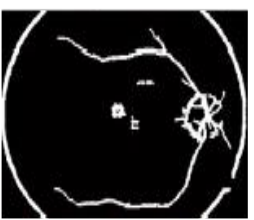

(d)
Fig.8. Segmentation Results (a) Segmentation image (b) Microaneurysms, Hemorrhages (c) Exudates (d) Blood Vessels

Further, several kinds of features such as texture features, shape features and statistical features are extracted. These extracted features are reducing improper classification and also 
improving accuracy rate. Finally, hybrid classifier is used for classifying the DR into multistage based on their severity. The severity levels are considered as normal, mild, moderate, severe and PDR as shown in Fig.9.

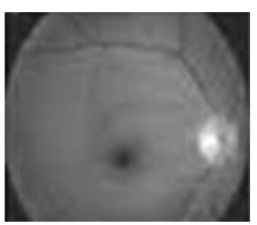

(a)

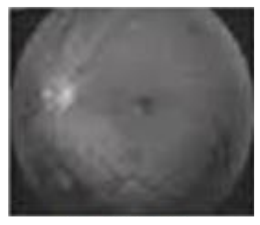

(c)

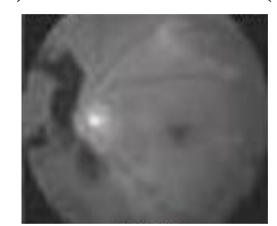

(e)

Fig.9. Multi Stage Classification of DR (a) Normal (b) Mild (c) Moderate (d) Severe (e) PDR

\subsection{COMPARATIVE ANALYSIS}

The proposed work is compared with other existing classification algorithms and techniques while concentrating on significant performance metrics.

\subsubsection{Accuracy:}

The proposed hybrid classification technique is compared with existing methods [6] [15] [17]. The obtained result is checked for accuracy which provides best result (almost 100\%) when compared with existing approaches. The Fig.10 illustrates the graphical result of classification approaches.

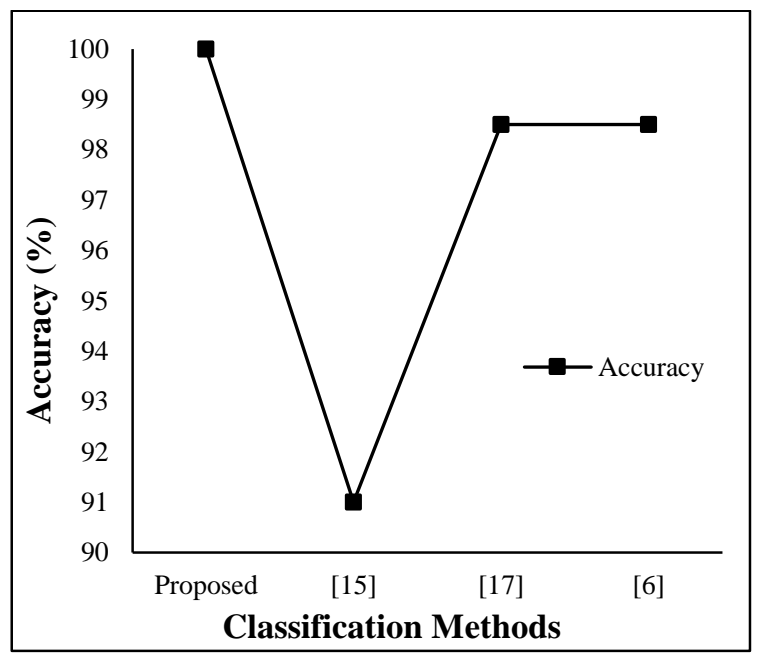

Fig.10. Accuracy of Various Classification Methods

\subsubsection{Error Rate:}

The proposed average error rate is compared with existing approach [17]. Commonly error occurs during classification, Fig.11 shows average error rate which is low in the proposed work.

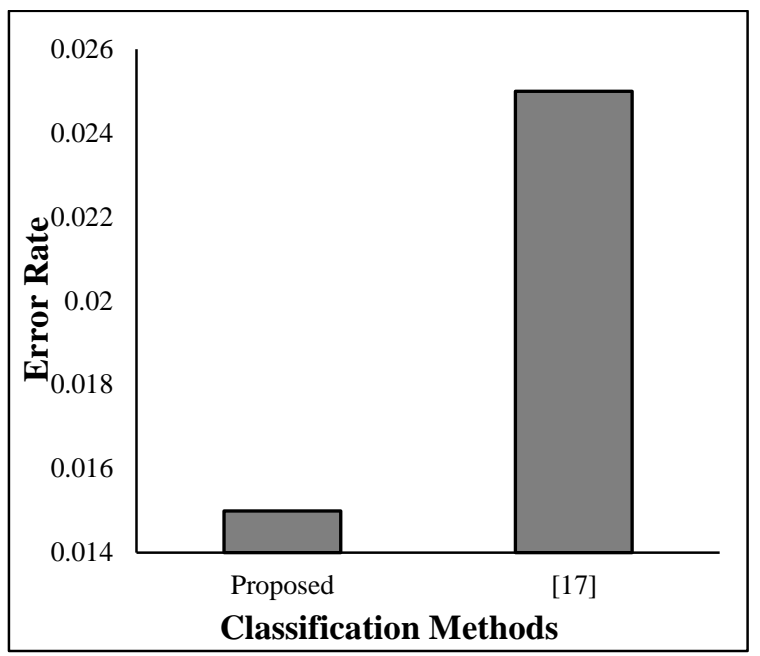

Fig.11. Average Error Rate

The Table. 3 represents the comparison result of accuracy, sensitivity and specificity. The proposed sensitivity and specificity result provides best accuracy result which clearly shown in Table.3. The proposed result is compared with existing results.

Table.3. Comparison of Sensitivity, Specificity and Accuracy

\begin{tabular}{|c|c|c|c|}
\hline Methods & Sensitivity & Specificity & Accuracy \\
\hline $\begin{array}{c}\text { Classification } \\
\text { techniques [6] }\end{array}$ & 98.6 & 97.2 & 98.4 \\
\hline $\begin{array}{c}\text { Machine Learning } \\
\text { Based Automatic } \\
\text { Detection[7] }\end{array}$ & 92.90 & 96.30 & 95.23 \\
\hline SVM [9] & 92 & 80 & 90 \\
\hline DREAM [12] & 100 & 53.16 & 90.4 \\
\hline $\begin{array}{c}\text { Automatic } \\
\text { screening [13] }\end{array}$ & 71 & 95 & 82 \\
\hline $\begin{array}{c}\text { Projection based } \\
\text { Algorithm[16] }\end{array}$ & 70 & 99.98 & 84 \\
\hline $\begin{array}{c}\text { Proposed Neuro- } \\
\text { Fuzzy }\end{array}$ & 100 & 100 & 100 \\
\hline \multicolumn{2}{|c|}{}
\end{tabular}

\section{CONCLUSION}

The proposed multistage DR classification of retinal fundus image is processed by pre-processing, segmentation, feature extraction and classification steps. Firstly, pre-processing of input image reduces noise level and also performed with contrast enhancement. Secondly, retinal structures are effectively segmented by using intensity operations. Thirdly, significant features are extracted with the support of texture, shape and statistical features. Neuro-fuzzy classifier is used for accurate classification of DR into multistage. The proposed hybrid 
classification result improves the overall accuracy level of multistage DR. The proposed process detects the DR disease in earlier stage which supports for medical treatment process. Additional features may be added as a future enhancement for detecting the DR disease. Then, feature selection process can also be added for selecting the best features which enhance the classification process.

\section{REFERENCES}

[1] Diabetic Retinopathy: Classification and Clinical Features, Available at: https://www.uptodate.com/contents/diabeticretinopathy-classification-and-clinical-features

[2] Vision 2020: The Right to Sight, Available at: https://www.iapb.org/vision-2020/

[3] Mohammed Shafeeq Ahmed and B. Indira, "A Survey on Automatic Detection of Diabetic Retinopathy", International Journal of Computer Engineering and Technology, Vol. 6, No. 11, pp. 36-45, 2015.

[4] A.P. Shingade and A.R. Kasetwar, "A Review on Implementation of Algorithms for Detection of Diabetic Retinopathy", International Journal of Research in Engineering and Technology, Vol. 3, No. 3, pp. 87-94, 2014.

[5] Jonathan Goh, Lilian Tang, George Saleh, Lutfiah Al turk, $\mathrm{Yu} \mathrm{Fu}$ and Antony Browne, "Filtering Normal Retinal Images for Diabetic Retinopathy Screening using Multiple Classifiers", Proceedings of IEEE International Conference on Information Technology and Applications in Biomedicine, pp. 220-228, 2009.

[6] Arslan Ahmad, Atif Bin Mansoor, Rafia Mumtaz, Mukaram Khan and S.H. Mirza, "Image Processing and Classification in Diabetic Retinopathy: A Survey", Proceedings of IEEE $5^{\text {th }}$ European Workshop in Visual Information Processing, pp. 1-6, 2014.

[7] Shuang Yu, Di Xiao and Yogesan Kanagasingam, "Machine Learning Based Automatic Neovascularization Detection on Optic Disc Region", IEEE Journal of Biomedical and Health Information, Vol. 22, No. 3, pp. 886-894, 2016.

[8] W. Kusakunniran, J. Rattanachoosin, K. Sutassananon and P. Anekkitphanich, "Automatic Quality Assessment and Segmentation of Diabetic Retinopathy Images", Proceedings of IEEE Region 10 Conference, pp. 997-1000, 2016.

[9] C. Aravind, M. Ponni Bala and S. Vijayachitra, "Automatic Detection of Microaneurysms and Classification of Diabetic Retinopathy Images using SVM Technique", Proceedings of International Conference on Innovations in Intelligent
Instrumentation, Optimization and Signal Processing, pp. 18-22, 2013

[10] Zahira Asifa Taranum and B. Srilatha, "Detection of Diabetic Retinopathy with feature extraction using Image processing", International Journal of Electrical and Computer Systems, Vol. 3, No. 8, pp. 1-4, 2015.

[11] Jaykurnar Lachure, A.V. Deorankar, Sagar Lachure, Swati Gupta and Romit Jadhav, "Diabetic Retinopathy using Morphological Operations and Machine Learning", Proceedings of IEEE International Advance Computing Conference, pp. 617-622, 2015.

[12] Sohini Roychowdhury and Dara D. Koozekanani, "DREAM: Diabetic Retinopathy Analysis using Machine Learning", IEEE Journal of Biomedical and Health Informatics, Vol. 18, No. 5, pp. 1-12, 2013.

[13] Sarni Suhaila Rahim, Vasile Palade, James Shuttleworth and Chrisina Jayne, "Automatic Screening and Classification of Diabetic Retinopathy and Maculopathy using Fuzzy Image Processing", Brain Informatics, Vol. 3, No. 4, pp. 249-267, 2016.

[14] Rubeena Banu, Vanishri Arun and N. Shankaraiah, "Metacognitive Neural Network Method for Classification of Diabetic Retinal Images", Proceedings of IEEE $2^{\text {nd }}$ International Conference on Cognitive Computing and Information Processing, pp. 1-5, 2016.

[15] Arash Razbon, Keyvan Mahjoory and Mahdi Nooshyar, "Segmentation of Retinal Blood Vessels by means of Gabor Wavelet and Fuzzy Mathematical Morphology", Proceedings of IEEE $2^{\text {nd }}$ International Conference on Signal Processing and Intelligent Systems, pp. 1-5, 2016.

[16] C. Eswaran, Marwan D. Saleh and Junaidi Abdullah, "Projection based Algorithm for Detecting Exudates in Color Fundus Images", Proceedings of IEEE $19^{\text {th }}$ International Conference on Digital Signal processing, pp. 459-463, 2014.

[17] A. Manjot Kaur and Parabh Preetkaur, "An Integrated Approach for Diabetic Retinopathy Exudate Segmentation by using Genetic Algorithm and Switching Median Filter", Proceedings of IEEE International Conference on Image, Vision and Computing, pp. 119- 123, 2016.

[18] Romany F. Mansour, "Evolutionary Computing Enriched Computer Aided Diagnosis System for Diabetic Retinopathy: A Survey", IEEE Reviews in Biomedical Engineering, Vol. 10, pp. 334-349, 2017.

[19] Muhammed Zubair and Hashim Ali, "Automated Segmentation of Hard Exudates using Dynamic Thresholding to Detect Diabetic Retinopathy in Retinal Photographs", Proceedings of International Conference on Innovative Computing, pp. 1-6, 2016. 